\title{
Monostability and Multistability of Genetic Regulatory Networks with Different Types of Regulation Functions
}

\author{
Wei Pan ${ }^{a, b}$, Zidong Wang ${ }^{c, *}$, Huijun $\mathrm{Gao}^{a}$ and Xiaohui $\mathrm{Liu}^{c}$
}

\begin{abstract}
Monostability and multistability are proven to be two important topics in synthesis biology and system biology. In this paper, both monostability and multistability are analyzed in a unified framework by applying control theory and mathematical tools. The genetic regulatory networks (GRNs) with multiple time-varying delays and different types of regulation functions are considered. By putting forward a general sector-like regulation function and utilizing up-to-date techniques, a novel Lyapunov-Krasovskii functional is introduced for achieving delay dependence to ensure less conservatism. A new condition is then proposed for the general stability of a GRN in the form of linear matrix inequalities (LMIs) that are dependent on the upper and lower bounds of the delays. Our general stability conditions are applicable to several frequently used regulation functions. It is shown that the existing results for monostability of GRNs are special cases of our main results. Five examples are employed to illustrate the applicability and usefulness of the developed theoretical results.
\end{abstract}

\section{Keywords}

Monostability, multistability, genetic regulatory networks, linear matrix inequality, multiple time-varying delay.

\section{INTRODUCTION}

In synthesis biology or system biology, the ultra goal is to engineer unnatural organic molecules that function in living systems to investigate natural biological phenomena and for a variety of applications [1-3]. In control engineering, the overall aim is to synthesize controllers which can achieve desired performance. Also, the notion of feedback, which is the core of control engineering, is a central recurring theme in the design of genetic regulatory networks (GRNs) [4,5]. In fact, feedback is so prevalent in biological systems that it can be found at all levels of organization, from the molecular and cellular levels, to the organism and ecological levels [6]. It is impossible to overstate the importance of feedback as a strategy for the maintenance and evolution of life. Therefore, it is reasonable to expect that ideas from control theory will lead to new understanding of the underlying biological processes [7].

Similar to other dynamical control systems, GRNs have the stability as their key property and stability analysis should be conducted prior to controller synthesis. Recall the concept of traditional stability that aims at the unique equilibrium point, we could name such a stability as monostability instead. Recently, a lot of efforts have been made to the monostability problem of GRNs. In [8], a simple genetic network model was studied to test the role of negative feedback in the monostability of GRNs. In [9], the monostability of GRNs with SUM-logic nonlinear regulatory functions of Hill type was studied. In [10], the robust asymptotic monostability of GRNs with time-varying delays and polytopic parameter uncertainties was studied. In [11],

This work was supported in part by the Biotechnology and Biological Sciences Research Council (BBSRC) of the U.K. under Grant BB/C506264/1, the Royal Society of the U.K., the National Natural Science Foundation of China under Grants 60504008 and 60804028, the Program for New Century Excellent Talents in Universities of China, and the Alexander von Humboldt Foundation of Germany.

${ }^{a}$ Space Control and Inertial Technology Research Center, Harbin Institute of Technology, Harbin 150001, China.

${ }^{b}$ Department of Electronic Science and Technology, University of Science and Technology of China, Hefei 230027, China.

${ }^{c}$ Department of Information Systems and Computing, Brunel University, Uxbridge, Middlesex, UB8 3PH, United Kingdom.

${ }^{*}$ Corresponding author. Email: Zidong.Wang@brunel.ac.uk. 
the discrete-time analog of the continuous-time GRN has been formulated and the monostability problem of discrete-time GRN with delays has been investigated. Other most recently published results can be found in [37-39] and the references therein.

The discovery of mathematical logic in gene regulation [12] laid a foundation for today's synthesis biology. As pointed out in [12], the co-repressive switches in the well-known lac operon in the bacteria Escherichia coli have long been proposed as a common regulatory theme, and the synthetic toggle switch $[4,13]$ serves as a model system in which multistability or bistability is the defining character to study such synthetic GRNs. The term 'multistability' aims at the coexistence of multiple steady states, which is different from monostability. Multistability, the capacity to achieve multiple internal states in response to a single set of external inputs, plays an important role in gene circuit design in synthesis biological systems. Multistability has certain unique properties which are not shared by other mechanisms of integrative control and it almost certainly plays an essential role in the dynamics of living cells and organisms [14,15]. The maintenance of phenotypic differences in the absence of genetic or environmental differences, which has been demonstrated experimentally for the regulation of the lactose operon in Escherichia coli, may be due to multistability. Cell differentiation might also be explained as multistability [16]. Bistability, the property of having two stable fixed points, is a basic case of multistability. It is becoming increasingly clear that bistability is an important recurring theme in cell signaling. Bistability is of particular relevance to biological systems that switch between discrete states, generating oscillatory responses.

Theoretical results obtained for the multistability of a GRN have been scattered in the literature. The biological system with multistability and hysteresis has been modeled as monotone dynamic systems in [17], where the rich and elegant theory of monotone dynamic system has provided an efficient mathematical tool for analysis (see [18] and references therein). Especially, in the biological systems with bistability, each stable mode of operation is associated with an appropriate invariant set in the state space and stability with respect to each set has been studied in terms of a local notion of input-to-state stability with respect to compact sets [19]. A general method for studying multistability in a large class of biological systems has been provided in [20]. Meanwhile, a piecewise power-law approximation has been proposed to approach bistability in [21] where the $S$-system models have been applied.

In this paper, either monostability or multistability of GRNs is referred to as general stability. It should be pointed out that, although the general stability of GRNs has received some initial research attention, there are still many open problems left for further investigation, three of which are listed as follows that motivate our current study:

- First, it has been recognized that the slow processes of transcription, translation and diffusion to the place of action of a protein inevitably cause time delays, which should be taken into account in the biological systems or artificial genetic networks in order to have more accurate models. Also, for different metabolites in GRNs, time delays of biochemical reactions may be various due to the different reaction pathways. However, the incorporation of multiple time-delays gives rise to significant difficulties in quantitative analysis of dynamics of GRNs.

- Second, the types of regulation functions employed in the literature are diverse, most of which are nonlinear $[22,23]$. In [24], a piecewise-multiaffine model is proposed to deal with the notoriously difficult analysis of general nonlinear systems, but that regulation functions are assumed to be precisely known as one type. In the presence of different types of regulation functions, how to develop a unified framework to analyze monostability or multistability of GRNs brings great challenges.

- Third, monostability is a special case of multistability, so monostability and multistability of GRNs should be investigated as a whole. This way, the existing results on monostability can be covered as special cases. How to construct a general stability condition to test both monostability and multistability is also of great interest. 
To sum up, it is essential and important to investigate the general stability of delayed GRNs with different types of regulation functions. Unfortunately, to the best of the authors' knowledge, up to now, very little effort has been made towards the above challenging problems, which then constitute the main focus of our present research.

In this paper, we are concerned with the general stability of GRNs with multiple time-varying delays. Several different types of regulation functions are considered. We introduce a novel Lyapunov-Krasovskii functional by utilizing the most updated techniques for achieving delay dependence. A new condition is then proposed for the general stability of a GRN with multiple time-varying delays and different types of regulation functions in the form of linear matrix inequalities (LMIs) that can be readily verified by using standard numerical software (such as Matlab) [25]. An important feature with the results to be reported is that, all the general stability conditions are dependent on the upper and lower bounds of the delays, which is made possible by utilizing the up-to-date techniques to achieve delay dependence. Second, our general stability conditions are applicable to several different regulation functions, which covers many types of currently investigated GRNs. Third, monostability and multistability analysis are investigated in a general framework, and the previous results on monostability of GRNs are included as special cases. Five examples are employed to illustrate the applicability and usefulness of the developed theoretical results. Example 1 is concerned with a constructed GRN with five equilibrium points of which three are stable. Example 2 deals with a model of transcription factors (TFs) subjected to its own transcription regulation which is displaying bistability. Example 3 tackles a synthetic, bistable genetic toggle switch in Escherichia coli. A GRN with five nodes is considered in Example 4 and a synthetic oscillatory network of transcriptional regulators is investigated in Example 5. It's shown that the existing results with respect to Example 4 and Example 5 are special cases of our results.

Notation: The notation used throughout the paper is standard. The superscript $T$ indicates matrix transposition; $\mathbb{R}^{n}$ denotes the $n$-dimensional Euclidean space and $\mathbb{R}^{n \times k}$ is the set of all $n \times k$ real matrices. $A_{n, k}$ denotes $A \in \mathbb{R}^{n \times k} . I$ and $\mathbf{0}$ denote identity matrix and zero matrix respectively, the notation $P>0$ means that $P$ is symmetric and positive definite and the symbol $*$ indicates symmetric blocks in the LMIs. In addition, $\operatorname{diag}\{\ldots\}$ stands for a block-diagonal matrix and for a matrix $A, \operatorname{sym}(\mathrm{A}) \operatorname{denotes} A+A^{T}$.

\section{Model ANd Preliminaries}

In this section, we introduce a GRN model described by the following differential equations for $i=1,2, \ldots, n$ :

$$
\dot{z}_{i}(t)=-a_{i} z_{i}(t)+R_{i}\left(z\left(t-h_{i}(t)\right)\right),
$$

where $z_{1}, \ldots, z_{n}$ are metabolites, such as genes, proteins, activators, repressors, enzymes, factors or products of a biochemical network, and $z(\cdot)=\left[z_{1}(\cdot), z_{2}(\cdot), \ldots, z_{n}(\cdot)\right]^{T} \in \mathbb{R}^{n}$ is the metabolites state vector. Their rates of degradation are denoted by $a_{i} \in R^{+}$. $\dot{z}_{i}$, the rate of change in $z_{i}$, represents concentration change of a variable due to production or degradation. $R_{i}(\cdot)$ represents the feedback regulation function on the $i$ th metabolite, which is generally a nonlinear or linear function on the variables $\left[z_{1}(\cdot), z_{2}(\cdot), \ldots, z_{n}(\cdot)\right]$, but has a form of monotonicity with each variable. Regulation functions are used to capture the combined effect of several regulatory proteins on the control of gene expression or protein degradation and it describes the connection and topology structure of metabolites. We assumed that

$$
\begin{aligned}
h_{i 1} & \leq h_{i}(t) \leq h_{i 2}, \\
\dot{h}_{i}(t) & \leq \mu_{i}<\infty .
\end{aligned}
$$

The regulation function $R_{i}(z(\cdot))$ is taken as

$$
R_{i}(z(\cdot))=\sum_{j=1}^{n} \alpha_{i j} R_{i j}\left(z_{j}(\cdot)\right)
$$


because each metabolite acts additively to regulate the $i$ th metabolite [26].

In the following, we will consider three special regulation functions in three remarks in order to show the existence of multiple equilibrium point, hence the multistability.

Remark 1: We consider a simplified GRN model:

$$
\dot{z}_{i}(t)=-a_{i} z_{i}(t)+\sum_{j=1}^{n} b_{i j} R_{i j}\left(z_{j}(t)\right) .
$$

A regulation function that is often found in the literature is the Hill type [23]:

$$
R_{i j}\left(z_{j}\right)=\frac{z_{j}^{H_{i j}}(t)}{\theta_{j}^{H_{i j}}+z_{j}^{H_{i j}}(t)},
$$

with $\theta_{j}>0$ the threshold for the regulatory influence of $z_{j}$ on a target metabolite $z_{i}$, and $H_{i j}$ is the Hill coefficients. The function ranges from 0 to 1 and increases as $z_{j} \rightarrow \infty$, so that an increase in $z_{j}$ will tend to increase the expression rate of the metabolite, then metabolite $j$ is an activator of metabolite $i$. If activator $j$ is an repressor of activator $i$, then

$$
R_{i j}\left(z_{j}\right)=1-\frac{z_{j}^{H_{i j}}(t)}{\theta_{j}^{H_{i j}}+z_{j}^{H_{i j}}(t)} .
$$

If $\theta_{j}=1$, the regulation function is in a standard form. (4) and (5) can be easily transformed to standard form. Then the GRN (3) can be rewritten as

$$
\dot{z}_{i}(t)=-a_{i} z_{i}(t)+\sum_{j=1}^{n} b_{i j} \bar{R}_{i j}\left(z_{j}(t)\right)+u_{i},
$$

for $i=1,2, \ldots, n$, with

$$
\bar{R}_{i j}\left(z_{j}(t)\right)=\frac{z_{j}^{H_{i j}}(t)}{\theta_{j}^{H_{i j}}+z_{j}^{H_{i j}}(t)}, u_{i}=\sum_{j \in \mathcal{F}_{i}} \alpha_{i j},
$$

where $\mathcal{F}_{i}$ is the set of all the $j$ which is a repressor of activator $i, u_{i}$ is defined as a basal rate. And if $b_{i j}=\alpha_{i j}$, $z_{j}$ is an activator of $z_{i}$; if $b_{i j}=0, z_{j}$ is no link with $z_{i}$; if $b_{i j}=-\alpha_{i j}, z_{j}$ is an a repressor of $z_{i}$.

We let $\dot{z}_{i}(t)=0$, that is, $a_{i} z_{i}(t)-u_{i}=\sum_{j=1}^{n} b_{i j} \bar{R}_{i j}\left(z_{j}(t)\right)$, the solution of this equation defines the equilibrium point. For convenience, we consider the one variable case and let $f(z)=z^{H_{i j}} /\left(1+z^{H_{i j}}\right), h(z)=a z-u$. It is shown that there could be three cross points at most when $H_{i j}=2$ in Fig. 1(a) and two cross points when $H_{i j}=1$ in Fig. 1(b).

Remark 2: A regulation function could also take the neural activation function type. Regulation functions of this type have continuous sigmoid curves, which can regulate the expression of switch-like behavior of genes. In a GRN, if metabolite $j$ is an activator of metabolite $i$, then

$$
R_{i j}\left(z_{j}\right)=\tanh \left(z_{j}\right)
$$

and if metabolite $j$ is an repressor of metabolite $i$,then

$$
R_{i j}\left(z_{j}\right)=-\tanh \left(z_{j}\right)
$$


(a)

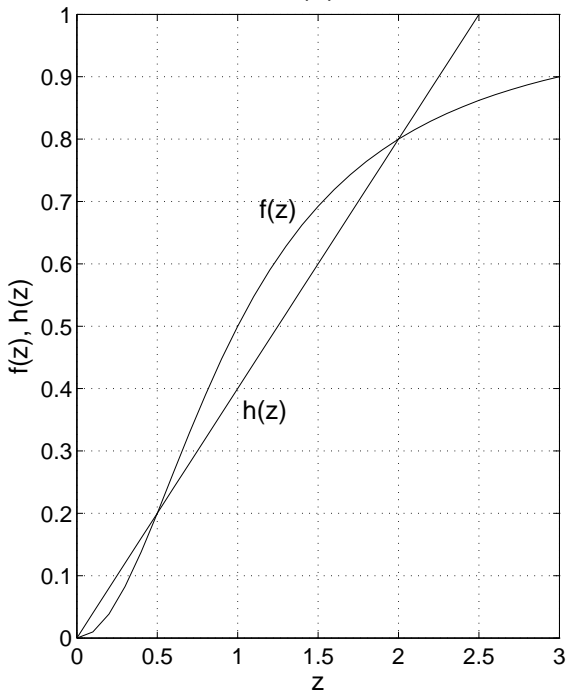

(b)

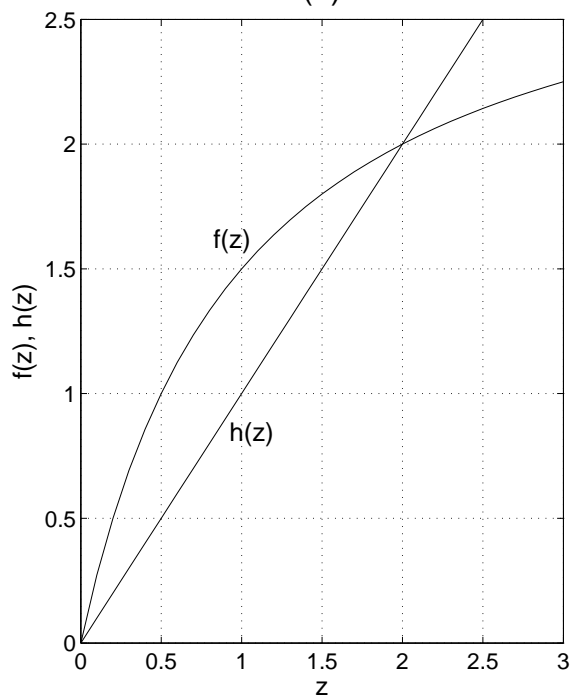

Fig. 1. (a) $f(z)=z^{2} /\left(1+z^{2}\right)$ and $h(z)=0.4 z$. Three equilibrium points can be achieved. (b) $f(z)=z /(1+z)$ and $h(z)=z$. Two equilibrium points can be obtained.

It's shown that the regulation function has a " $S$ " shape and there are three cross points at most in Fig. 2(a). To study multistability of GRNs, here we propose a new form of regulation function, if metabolite $j$ is an activator of metabolite $i$, then

$$
R_{i j}\left(z_{j}\right)=\tanh \left(z_{j}^{3}\right)
$$

and if metabolite $j$ is an repressor of metabolite $i$, then

$$
R_{i j}\left(z_{j}\right)=-\tanh \left(z_{j}^{3}\right) .
$$

For convenience, we consider the one-variable case and let $h(z)=z, f(z)=\tanh (z)$. It is shown that there could be three cross points at most in Fig. 2(a). Let $h(z)=0.7 z, f(z)=\tanh \left(z^{3}\right)$. It's shown that the regulation function has a double "S" shape and there are five cross points at most in Fig. 2(b). In fact, all the variables should be positive in GRNs, but by a shift, the variables of regulation functions here could be positive with the shape of regulation functions holding.

Remark 3: A regulation function could also take the straight line type [27,28]. In a GRN, if metabolite $j$ is an activator of metabolite $i$, then

$$
R_{i j}\left(z_{j}\right)=z_{j}
$$

and if metabolite $j$ is an repressor of metabolite $i$, then

$$
R_{i j}\left(z_{j}\right)=1-z_{j}
$$

Then we consider a GRN as follows:

$$
\dot{z}_{i}(t)=-a_{i} z_{i}(t)+\sum_{j=1}^{n} b_{i j} g_{i j}\left(z_{j}\left(t-h_{i}(t)\right)\right)+u_{i}
$$

where $i=1,2, \ldots, n, u_{i}$ is the basal rate and the regulation function $g_{i j}(\cdot)$ is described as follows. Each regulation function $g_{i j}\left(z_{j}\right)$ is divided into $k_{g i j \max }$ piecewise intervals along the variable $z_{j}$ :

$$
\bar{\Omega}_{g}=\left\{\begin{array}{c}
{\left[\lambda_{i j 0}, \lambda_{i j 1}\right]^{\delta_{i j 1}^{(i)}} \times\left[\lambda_{i j 1}, \lambda_{i j 2}\right]^{\delta_{i j 2}^{(i)}} \times \cdots \times\left[\lambda_{i j\left(k_{g i j \max }-1\right)}, \lambda_{i j k_{g i j \max }}\right]^{\delta_{i j k g i j \max }^{(i)}},} \\
\left(\delta_{i j 1}^{(i)}, \delta_{i j 2}^{(i)}, \ldots, \delta_{i j k_{g i j \max }}^{(i)}\right)=(1,0, \ldots, 0) \text { or }(0,1, \ldots, 0) \text { or }(0, \ldots 0,1), i=1,2, \ldots, n
\end{array}\right\} .
$$


(a)

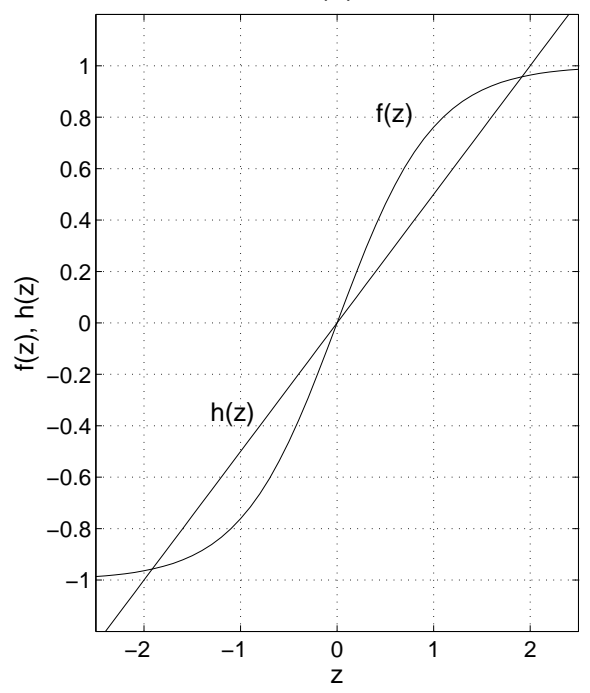

(b)

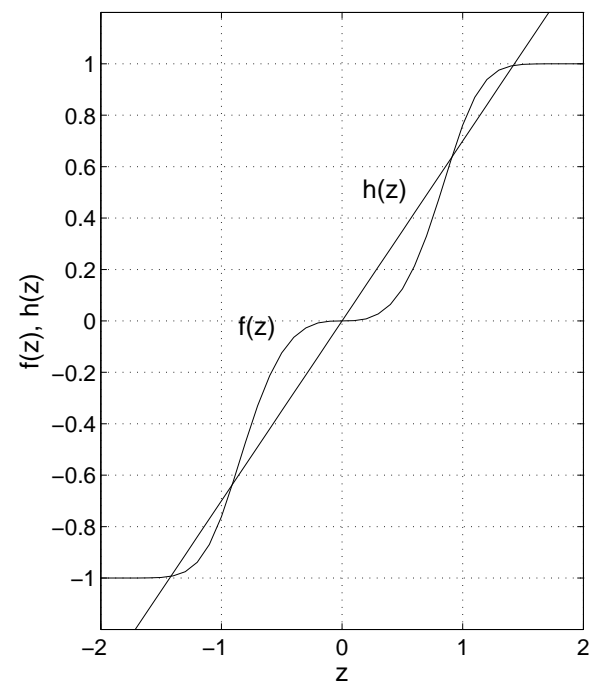

Fig. 2. (a) $h(z)=z$ and $f(z)=\tanh (z)$. Three equilibrium points can be achieved. (b) $h(z)=0.7 z$ and $f(z)=\tanh \left(z^{3}\right)$. Five equilibrium points can be obtained.

If there exist $N$ equilibrium points of the (13), let $k_{g i j \max }=N$. Select $N$ intervals

$$
\Omega_{g}=\left\{\begin{array}{l}
{\left[\lambda_{i j 0}, \lambda_{i j 1}\right]^{\delta_{i j 1}^{(i)}} \times\left[\lambda_{i j 1}, \lambda_{i j 2}\right]^{\delta_{i j 2}^{(i)}} \times \cdots \times\left[\lambda_{i j(N-1)}, \lambda_{i j N}\right]^{\delta_{i j N}^{(i)}}} \\
\left(\delta_{i j 1}^{(i)}, \delta_{i j 2}^{(i)}, \ldots, \delta_{i j N}^{(i)}\right)=(1,0, \ldots, 0) \text { or }(0,1, \ldots, 0) \text { or }(0, \ldots 0,1), i=1,2, \ldots, n
\end{array}\right\} .
$$

Then the $k$ th equilibrium point lies in this region

$$
\Omega_{g_{k}}=\left\{\prod_{i=1}^{n}\left[\cap \prod_{j=1}^{n}\left[\lambda_{i j(k-1)}, \lambda_{i j k}\right]\right]\right\} .
$$

Having demonstrated the multistability behavior of GRNs with different regulation functions, we now propose a general sector-like regulation function in order to facilitate the generality of our stability analysis.

Assumption 1: Let $k_{g i j \max }=N$. Each regulation function in $(13), g_{i j}(\cdot), i, j=1,2, \ldots, n$, satisfies the following condition when $\forall x_{k}, y_{k} \in\left[\lambda_{i j(k-1)}, \lambda_{i j k}\right], x_{k} \neq y_{k}, 1 \leq k \leq N$ :

$$
l_{k i j}^{-} \leq \frac{g_{i j}\left(x_{k}\right)-g_{i j}\left(y_{k}\right)}{x_{k}-y_{k}} \leq l_{k i j}^{+}
$$

where $l_{k i j}^{-}$and $l_{k i j}^{+}$are positive constants. Define diagonal matrices $L_{k i}^{-}=\operatorname{diag}\left\{\mathrm{l}_{\mathrm{ki} 1}^{-}, \ldots, \mathrm{l}_{\mathrm{kin}}^{-}\right\}$and $L_{k i}^{+}=$ $\operatorname{diag}\left\{\mathrm{l}_{\mathrm{ki} 1}^{+}, \ldots, \mathrm{l}_{\mathrm{kin}}^{+}\right\}$.

Remark 4: The inequality (14) is similar to the one proposed in [33-35] for the activation function of neural networks. As pointed out in [33,34], this description could be non-monotonic, and is more general than the usual sigmoid functions and the recently commonly used Lipschitz conditions. We like to point out that such a description is very precise/tight in quantifying the lower and upper bounds of the regulation functions, hence very helpful for using LMI-based approach to reduce the possible conservatism.

We assume that a GRN (13) has $N$ equilibrium points. Let $z_{k}^{*}=\left(z_{k 1}^{*}, z_{k 2}^{*}, \ldots, z_{k n}^{*}\right)^{T}$ be the $k$ th equilibrium point, $i=1,2, \cdots, N$. In the following, the equilibrium point $z_{k}^{*}=\left(z_{k 1}^{*}, z_{k 2}^{*}, \ldots, z_{k n}^{*}\right)^{T}$ of system (13) is firstly shifted to origin by the transformation $x(\cdot)=z(\cdot)-z_{k}^{*}$, thus we have the following form:

$$
\dot{x}_{i}(t)=-a_{i} x_{i}(t)+\sum_{j=1}^{n} b_{i j} f_{k i j}\left(x_{j}\left(t-h_{i}(t)\right)\right)
$$


where

$$
f_{k i j}\left(x_{j}(\cdot)\right)=g_{i j}\left(x_{j}(\cdot)+z_{k j}^{*}\right)-g_{i j}\left(z_{k j}^{*}\right) .
$$

By Assumption 1 and (16), it is not difficult to verify that

$$
l_{k i j}^{-} \leq \frac{f_{k i j}\left(x_{j}\right)}{x_{j}} \leq l_{k i j}^{+}, \forall x_{j} \neq 0, \quad i=1,2, \ldots, n,
$$

and it is easy to see that $f_{k i j}(0)=0$.

Equivalently, (15) can be written as

$$
\dot{x}(t)=-A x(t)+\sum_{i=1}^{n} B_{i} f_{k i}\left(x\left(t-h_{i}(t)\right)\right)
$$

with $h_{1 i} \leq h(t) \leq h_{2 i}, \dot{h}_{i}(t) \leq \mu_{i}<\infty$, and

$$
f_{k i}\left(x\left(t-h_{i}(t)\right)\right)=\left[\begin{array}{c}
f_{k i 1}\left(x_{1}\left(t-h_{i}(t)\right)\right) \\
f_{k i 2}\left(x_{2}\left(t-h_{i}(t)\right)\right) \\
\vdots \\
f_{k i n}\left(x_{n}\left(t-h_{i}(t)\right)\right)
\end{array}\right],
$$

and

$$
B_{1}=\left[\begin{array}{c}
b_{1} \\
0 \\
\vdots \\
0
\end{array}\right], B_{2}=\left[\begin{array}{c}
0 \\
b_{2} \\
\vdots \\
0
\end{array}\right], \ldots, B_{n}=\left[\begin{array}{c}
0 \\
0 \\
\vdots \\
b_{n}
\end{array}\right],
$$

with $b_{i}=\left[b_{i 1}, b_{i 2}, \ldots, b_{i n}\right], i=1,2, \ldots, n$.

\section{Monostability and Multistability Conditions of GRNs}

In this section, we present our new delay-dependent general stability condition for the a GRN with multiple time-varying delays described in the previous section.

Definition 1: A GRN is said to have $N$-stability if it has $N(N \geq 1)$ stable equilibrium points. In this case, the GRN is said to be $N$-stable. Specially, we name monostability of a GRN if $N=1$ and bistability of a GRN if $N=2$.

Theorem 1: Let $O=\left[0_{n}, 0_{n}, \ldots, 0_{n}\right]_{n, n n}$ and $B=\left[B_{1}, B_{2}, \ldots, B_{n}\right]_{n, n n}$. The system in (13) is asymptotically $N$-stable if there exist matrices $P_{k}>0, Q_{k j i}>0, Z_{k j i}>0$, and positive diagonal matrices $S_{k i}^{+}$and $S_{k i}^{-}$ $(k=1, \cdots, N, i=1, \cdots, n, j=1,2)$ such that the following LMIs hold:

$$
\Theta_{k}=W_{k P}^{T} \bar{P}_{k} W_{k P}+\sum_{i=1}^{n}\left(W_{k Q_{i}}^{T} \bar{Q}_{k i} W_{k Q_{i}}+W_{k Z_{i}}^{T} \bar{Z}_{k i} W_{k Z_{i}}+W_{k S_{i}^{+}}^{T} \bar{S}_{k i}^{+} W_{k S_{i}^{+}}+W_{k S_{i}^{-}}^{T} \bar{S}_{k i}^{-} W_{k S_{i}^{-}}\right)<0
$$


where

$$
\begin{aligned}
& \bar{Q}_{k}=\operatorname{diag}\left\{\mathrm{Q}_{\mathrm{k} 1 \mathrm{i}},-\mathrm{Q}_{\mathrm{k} 1 \mathrm{i}}, \mathrm{Q}_{\mathrm{k} 2 \mathrm{i}},-\left(1-\mu_{\mathrm{i}}\right) \mathrm{Q}_{\mathrm{k} 2 \mathrm{i}}\right\}, \overline{\mathrm{Z}}_{\mathrm{k}}=\operatorname{diag}\left\{\mathrm{Z}_{\mathrm{k} 1 \mathrm{i}}, \mathrm{Z}_{\mathrm{k} 2 \mathrm{i}},-\mathrm{Z}_{\mathrm{k} 1 \mathrm{i}},-\mathrm{Z}_{\mathrm{k} 2 \mathrm{i}}\right\}, \\
& \bar{P}_{k}=\left[\begin{array}{cc}
0_{n} & P_{k} \\
P_{k} & 0_{n}
\end{array}\right], \bar{S}_{k i}^{+}=\left[\begin{array}{cc}
0_{n} & S_{k i}^{+} \\
S_{k i}^{+} & -S_{k i}^{+}
\end{array}\right], \bar{S}_{k i}^{-}=\left[\begin{array}{cc}
0_{n} & S_{k i}^{-} \\
S_{k i}^{-} & -S_{k i}^{-}
\end{array}\right],
\end{aligned}
$$

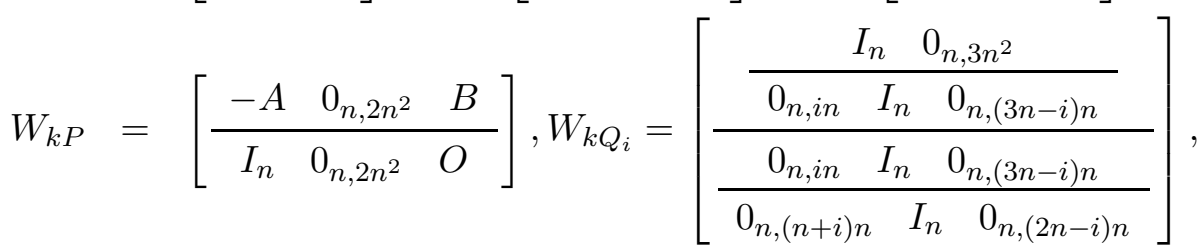

$$
\begin{aligned}
& W_{k Z_{i}}=\left[\begin{array}{rllll}
-\sqrt{h_{1 i}} A & 0_{n,(2 n+i-1) n} & \sqrt{h_{1 i}} B_{i} & 0_{n,(n-i) n} \\
\cline { 2 - 4 } & -\sqrt{h_{2 i}-h_{1 i}} A & 0_{n,(2 n+i-1) n} & \sqrt{h_{2 i}-h_{1 i}} B_{i} & 0_{n,(n-i) n} \\
\hline \sqrt{1 / h_{1 i}} I_{n} & 0_{n,(i-1) n} & -\sqrt{1 / h_{1 i}} I_{n} & 0_{n,(3 n-i) n} \\
\hline 0_{n, i n} & \sqrt{1 /\left(h_{2 i}-h_{1 i}\right)} I_{n} & 0_{n,(n-1) n} & -\sqrt{1 /\left(h_{2 i}-h_{1 i}\right)} I_{n} & 0_{n,(2 n-i) n}
\end{array}\right], \\
& W_{k S_{i}^{+}}=\left[\begin{array}{ccc}
0_{n,(n+i) n} & \sqrt{1 / 2} L_{k i}^{+} & 0_{n,(2 n-i) n} \\
\hline 0_{n,(2 n+i) n} & \sqrt{2} I_{n} & 0_{n,(n-i) n}
\end{array}\right], W_{k S_{i}^{-}}=\left[\begin{array}{ccc}
0_{n,(n+i) n} & -\sqrt{1 / 2} L_{k i}^{-} & 0_{n,(2 n-i) n} \\
\hline 0_{n,(2 n+i) n} & -\sqrt{2} I_{n} & 0_{n,(n-i) n}
\end{array}\right] .
\end{aligned}
$$

Proof: Let us show the $k$ th equilibrium point is asymptotically stable. The Lyapunov-Krasovskii functional is defined as follows:

$$
\begin{aligned}
V_{k}(x(t)) & =V_{k 1}(x(t))+V_{k 2}(x(t))+V_{k 3}(x(t)), \\
V_{k 1}(x(t)) & =x^{T}(t) P_{k} x(t), \\
V_{k 2}(x(t)) & =\sum_{i=1}^{n}\left[\int_{t-h_{1 i}}^{t} x^{T}(\alpha) Q_{k 1 i} x(\alpha) d \alpha+\int_{t-h_{i}(t)}^{t-h_{1 i}} x^{T}(\alpha) Q_{k 2 i} x(\alpha) d \alpha\right], \\
V_{k 3}(x(t)) & =\sum_{i=1}^{n}\left[\int_{-h_{1 i}}^{0} \int_{t+\beta}^{t} \dot{x}^{T}(\alpha) Z_{k 1 i} \dot{x}(\alpha) d \alpha d \beta+\int_{-h_{2 i}}^{-h_{1 i}} \int_{t+\beta}^{t} \dot{x}^{T}(\alpha) Z_{k 2 i} \dot{x}(\alpha) d \alpha d \beta\right] .
\end{aligned}
$$

The derivatives of $V_{k j}(x(t)), j=1,2,3$, are given by

$$
\begin{aligned}
\dot{V}_{k 1}(x(t))= & 2 x^{T}(t) P_{k} \dot{x}(t), \\
\dot{V}_{k 2}(x(t))= & \sum_{i=1}^{n}\left[x^{T}(t) Q_{k 1 i} x(t)-x^{T}\left(t-h_{1 i}\right) Q_{k 1 i} x\left(t-h_{1 i}\right)+x^{T}\left(t-h_{1 i}\right) Q_{k 2 i} x\left(t-h_{1 i}\right)\right. \\
& \left.-\left(1-\dot{h}_{i}(t)\right) x^{T}\left(t-h_{i}(t)\right) Q_{k 2 i} x\left(t-h_{i}(t)\right)\right] \\
\leq & \sum_{i=1}^{n}\left[x^{T}(t) Q_{k 1 i} x(t)-x^{T}\left(t-h_{1 i}\right) Q_{k 1 i} x\left(t-h_{1 i}\right)+x^{T}\left(t-h_{1 i}\right) Q_{k 2 i} x\left(t-h_{1 i}\right)\right. \\
& \left.-\left(1-\mu_{i}\right) x^{T}\left(t-h_{i}(t)\right) Q_{k 2 i} x\left(t-h_{i}(t)\right)\right] \\
\dot{V}_{k 3}(x(t))= & \sum_{i=1}^{n}\left[\dot{x}^{T}(t)\left(h_{1 i} Z_{k 1 i}+\left(h_{2 i}-h_{1 i}\right) Z_{k 2 i}\right) \dot{x}(t)\right. \\
& \left.-\int_{t-h_{1 i}}^{t} \dot{x}^{T}(\alpha) Z_{k 1 i} \dot{x}(\alpha) d \alpha-\int_{t-h_{2 i}}^{t-h_{1 i}} \dot{x}^{T}(\alpha) Z_{k 2 i} \dot{x}(\alpha) d \alpha\right] .
\end{aligned}
$$


From Jensen's inequality, we can easily get

$$
\begin{aligned}
& -\int_{t-h_{1 i}}^{t} \dot{x}^{T}(\alpha) Z_{k 1 i} \dot{x}(\alpha) d \alpha \\
\leq & -\frac{1}{h_{1 i}}\left[\int_{t-h_{1 i}}^{t} \dot{x}(\alpha) d \alpha^{T}\right]^{T} Z_{k 1 i}\left[\int_{t-h_{1 i}}^{t} \dot{x}(\alpha) d \alpha\right] \\
= & -\frac{1}{h_{1 i}}\left[x(t)-x\left(t-h_{1 i}\right)\right]^{T} Z_{k 1_{i}}\left[x(t)-x\left(t-h_{1 i}\right)\right], \\
& -\int_{t-h_{2 i}}^{t-h_{1 i}} \dot{x}^{T}(\alpha) Z_{k 2 i} \dot{x}(\alpha) d \alpha \\
\leq & -\int_{t-h_{i}(t)}^{t-h_{1 i}} \dot{x}^{T}(\alpha) Z_{k 2 i} \dot{x}(\alpha) d \alpha \\
\leq & -\frac{1}{h_{2 i}-h_{1 i}}\left[\int_{t-h_{i}(t)}^{t-h_{1 i}} \dot{x}(\alpha) d \alpha\right]^{T} Z_{k 2 i}\left[\int_{t-h_{i}(t)}^{t-h_{1 i}} \dot{x}(\alpha) d \alpha\right] \\
\leq & -\frac{1}{h_{2 i}-h_{1 i}}\left[x\left(t-h_{1 i}\right)-x\left(t-h_{i}(t)\right)\right]^{T} Z_{k 2 i} \\
& \times\left[x\left(t-h_{1 i}\right)-x\left(t-h_{i}(t)\right)\right] .
\end{aligned}
$$

By (17), for any scalar $s_{k i j}^{+} \geq 0$, it is clear that for $i, j=1, \cdots, n$.

$$
2 \sum_{j=1}^{n} s_{k i j}^{+} f_{k i j}\left(x_{j}\left(t-h_{i}(t)\right)\right)\left[l_{k i j}^{+} x_{j}\left(t-h_{i}(t)\right)-f_{k i j}\left(x_{j}\left(t-h_{i}(t)\right)\right)\right] \geq 0
$$

then

$$
\sum_{i=1}^{n} \sum_{j=1}^{n}\left[2 s_{k i j}^{+} f_{k i j}\left(x_{j}\left(t-h_{i}(t)\right)\right) l_{k i j}^{+} x_{j}\left(t-h_{i}(t)\right)-2 s_{k i j}^{+} f_{k i j}\left(x_{j}\left(t-h_{i}(t)\right)\right) f_{k i j}\left(x_{j}\left(t-h_{i}(t)\right)\right)\right] \geq 0,
$$

or equivalently

$$
\sum_{i=1}^{n}\left[2 f_{k i}\left(x\left(t-h_{i}(t)\right)\right) S_{k i}^{+} L_{k i}^{+} x\left(t-h_{i}(t)\right)-2 f_{k i}\left(x\left(t-h_{i}(t)\right)\right) S_{k i}^{+} f_{k i}\left(x\left(t-h_{i}(t)\right)\right)\right] \geq 0 .
$$

For any scalar $s_{k i j}^{-} \geq 0$, it is clear that for $i, j=1, \cdots, n$.

$$
2 \sum_{j=1}^{n} s_{k i j}^{-} f_{k i j}\left(x_{j}\left(t-h_{i}(t)\right)\right)\left[f_{k i j} h_{i j}\left(x_{j}\left(t-h_{i}(t)\right)\right)-l_{k i j}^{-} x_{j}\left(t-h_{i}(t)\right)\right] \geq 0
$$

then

$$
\sum_{i=1}^{n} \sum_{j=1}^{n}\left[2 s_{k i j}^{-} f_{k i j}\left(x_{j}\left(t-h_{i}(t)\right)\right) f_{k i j}\left(x_{j}\left(t-h_{i}(t)\right)\right)-2 s_{k i j}^{-} f_{k i j}\left(x_{j}\left(t-h_{i}(t)\right)\right) l_{k i j}^{-} x_{j}\left(t-h_{i}(t)\right)\right] \geq 0,
$$

or equivalently

$$
\sum_{i=1}^{n}\left[2 f_{k i}\left(x\left(t-h_{i}(t)\right)\right) S_{k i}^{-} f_{k i}\left(x\left(t-h_{i}(t)\right)\right)-2 f_{k i}\left(x\left(t-h_{i}(t)\right)\right) S_{k i}^{-} L_{k i}^{-} x\left(t-h_{i}(t)\right)\right] \geq 0 .
$$


By using (18), (27), (28), (29) and (30), we have

$$
\begin{aligned}
& \dot{V}_{k}(x(t)) \\
\leq & 2 x^{T}(t) P_{k}\left[-A x(t)+\sum_{i=1}^{n} B_{i} f_{k i}\left(x\left(t-h_{i}(t)\right)\right]\right. \\
& +\sum_{i=1}^{n}\left[x^{T}(t) Q_{k 1 i} x(t)-x^{T}\left(t-h_{1 i}\right) Q_{k 1 i} x\left(t-h_{1 i}\right)+x^{T}\left(t-h_{1 i}\right) Q_{k 2 i} x\left(t-h_{1 i}\right)\right. \\
& \left.-\left(1-\mu_{i}\right) x^{T}\left(t-h_{i}(t)\right) Q_{k 2 i} x\left(t-h_{i}(t)\right)\right]+\sum_{i=1}^{n}\left\{\left[-A x(t)+\sum_{i=1}^{n} B_{i} f_{k i}\left(x\left(t-h_{i}(t)\right)\right]^{T}\right.\right. \\
& \times\left[h_{1 i} Z_{k 1 i}+\left(h_{2 i}-h_{1 i}\right) Z_{k 2 i}\right]\left[-A x(t)+\sum_{i=1}^{n} B_{i} f_{k i}\left(x\left(t-h_{i}(t)\right)\right]\right. \\
& -\frac{1}{h_{1 i}}\left[x(t)-x\left(t-h_{1 i}\right)\right]^{T} Z_{k 1 i}\left[x(t)-x\left(t-h_{1 i}\right)\right] \\
& \left.-\frac{1}{h_{2 i}-h_{1 i}}\left[x\left(t-h_{1 i}\right)-x\left(t-h_{i}(t)\right)\right]^{T} Z_{k 2 i}\left[x\left(t-h_{1 i}\right)-x\left(t-h_{i}(t)\right)\right]\right\} \\
& +\sum_{i=1}^{n}\left[2 f_{k i}\left(x\left(t-h_{i}(t)\right)\right) S_{k i}^{+} L_{k i}^{+} x\left(t-h_{i}(t)\right)-2 f_{k i}\left(x\left(t-h_{i}(t)\right)\right) S_{k i}^{+} f_{k i}\left(x\left(t-h_{i}(t)\right)\right)\right] \\
& +\sum_{i=1}^{n}\left[2 f_{k i}\left(x\left(t-h_{i}(t)\right)\right) S_{k i}^{-} f_{k i}\left(x\left(t-h_{i}(t)\right)\right)-2 f_{k i}\left(x\left(t-h_{i}(t)\right)\right) S_{k i}^{-} L_{k i}^{-} x\left(t-h_{i}(t)\right)\right] \\
= & \zeta_{k}^{T}(t) \Theta \Theta_{k} \zeta_{k}(t)
\end{aligned}
$$

where

$$
\zeta_{k}(t)=\left[\begin{array}{c}
x(t) \\
\chi\left(t-h_{1}\right) \\
\chi(t-h(t)) \\
f_{k}(x(t-h(t)))
\end{array}\right]
$$

with

$$
\chi\left(t-h_{1}\right)=\left[\begin{array}{c}
x\left(t-h_{11}\right) \\
x\left(t-h_{12}\right) \\
\vdots \\
x\left(t-h_{1 n}\right)
\end{array}\right], \chi(t-h(t))=\left[\begin{array}{c}
x\left(t-h_{1}(t)\right) \\
x\left(t-h_{2}(t)\right) \\
\vdots \\
x\left(t-h_{n}(t)\right)
\end{array}\right], f_{k}(x(t-h(t)))=\left[\begin{array}{c}
f_{k 1}\left(x\left(t-h_{1}(t)\right)\right) \\
f_{k 2}\left(x\left(t-h_{2}(t)\right)\right) \\
\vdots \\
f_{k n}\left(x\left(t-h_{n}(t)\right)\right)
\end{array}\right] .
$$

Therefore,

$$
\dot{V}_{k}(t) \leq \zeta_{k}^{T}(t) \Theta_{k} \zeta_{k}(t)
$$

and then we have

$$
\Theta_{k}<0 .
$$

Since (31) holds, we have $\dot{V}_{k}(x(t))<-\epsilon_{k}\|x(t)\|^{2}$ for a sufficiently small $\epsilon_{k}>0, k=1, \cdots, N$ and $x(t) \neq 0$, then the asymptotic $N$-stability is established and the GRN (13) is $N$-stable.

Remark 5: Time delays are frequently encountered in not only the biological networks but also many other practical engineering systems, such as communication, electronics, and chemical systems. In the past decade, monostability analysis and synthesis problems for various time-delay systems have gained considerable research interests and a large amount of results have appeared in the literature, see, e.g., [29-31]. Recently, the problem of delay-dependent stability analysis for time-delay systems has received much attention and fruitful results 
have been reported. For the case when time-varying delays are considered, corresponding results can be found in [32]. It is noticed that, up to now, most existing results are restrictive to some extent since they have assumed that either the derivatives of the delays are less than 1 (e.g., [9]). Therefore, one of the objectives in this theorem is to remove the restriction and reduce the possible conservatism by introducing an advanced parameter-dependent Lyapunov functional.

If time delay is assumed to be constant, then the GRN (13) becomes:

$$
\dot{z}_{i}(t)=-a_{i} z_{i}(t)+\sum_{j=1}^{n} b_{i j} g_{i j}\left(z_{j}\left(t-\tau_{i}\right)\right)+u_{i}
$$

For the $k$ th equilibrium point, by transformation we have

$$
\dot{x}(t)=-A x(t)+\sum_{i=1}^{n} B_{i} f_{k i}\left(x\left(t-\tau_{i}\right)\right),
$$

Based on Theorem 1, we can get the following corollary.

Corollary 1: Let $O=\left[0_{n}, 0_{n}, \ldots, 0_{n}\right]_{n, n n}$ and $B=\left[B_{1}, B_{2}, \ldots, B_{n}\right]_{n, n n}$. The system in (32) is asymptotically $N$-stable, if there exist matrices $P_{k}>0, Q_{k i}>0, Z_{k i}>0$, and diagonal matrices $S_{k i}^{+}$and $S_{k i}^{-}, k=1, \cdots, N$, $i=1, \cdots, n$, such that the following LMIs hold:

$$
\Theta_{k}=W_{P}^{T} \bar{P}_{k} W_{P}+\sum_{i=1}^{n}\left(W_{Q}^{T} \bar{Q}_{k} W_{Q}+W_{R}^{T} \bar{R}_{k} W_{R}+W_{k S^{+}}^{T} \bar{S}_{k i}^{+} W_{k S^{+}}+W_{k S^{-}}^{T} \bar{S}_{k i}^{-} W_{k S^{-}}\right)<0
$$

where

$$
\begin{aligned}
& \bar{Q}_{k}=\operatorname{diag}\left\{\mathrm{Q}_{\mathrm{ki}},-\mathrm{Q}_{\mathrm{ki}}\right\}, \overline{\mathrm{Z}}_{\mathrm{k}}=\operatorname{diag}\left\{\mathrm{Z}_{\mathrm{ki}},-\mathrm{Z}_{\mathrm{ki}},\right\}, \\
& \bar{P}_{k}=\left[\begin{array}{cc}
0_{n} & P_{k} \\
P_{k} & 0_{n}
\end{array}\right], \bar{S}_{k i}^{+}=\left[\begin{array}{cc}
0_{n} & S_{k i}^{+} \\
S_{k i}^{+} & -S_{k i}^{+}
\end{array}\right], \bar{S}_{k i}^{-}=\left[\begin{array}{cc}
0_{n} & S_{k i}^{-} \\
S_{k i}^{-} & -S_{k i}^{-}
\end{array}\right], \\
& W_{k P}=\left[\begin{array}{ccc}
-A & O & B \\
\hline I_{n} & O & O
\end{array}\right], W_{k Q_{i}}=\left[\begin{array}{ccc}
I_{n} & 0_{n, 2 n^{2}} \\
\hline 0_{n, i n} & I_{n} & 0_{n,(2 n-i) n}
\end{array}\right] \text {, } \\
& W_{k Z_{i}}=\left[\begin{array}{ccccc}
-\sqrt{\tau_{i}} A & O & \sqrt{\tau_{i}} B & \\
\hline \sqrt{1 / \tau_{i}} I_{n} & 0_{n,(i-1) n} & -\sqrt{1 / \tau_{i}} I_{n} & 0_{n,(2 n-i) n}
\end{array}\right], \\
& W_{k S_{i}^{+}}=\left[\begin{array}{ccc}
0_{n, i n} & \sqrt{1 / 2} L_{k i}^{+} & 0_{n,(2 n-i) n} \\
\hline 0_{n,(n+i) n} & \sqrt{2} I_{n} & 0_{n,(n-i) n}
\end{array}\right], W_{k S_{i}^{-}}=\left[\begin{array}{ccc}
0_{n, i n} & -\sqrt{1 / 2} L_{k i}^{-} & 0_{n,(2 n-i) n} \\
\hline 0_{n,(n+i) n} & -\sqrt{2} I_{n} & 0_{n,(n-i) n}
\end{array}\right] .
\end{aligned}
$$

Furthermore, if the time delay is zero, then the GRN (13) becomes:

$$
\dot{z}_{i}(t)=-a_{i} z_{i}(t)+\sum_{j=1}^{n} b_{i j} g_{i j}\left(z_{j}(t)\right)+u_{i}
$$

For the $k$ th equilibrium point, by transformation we have

$$
\dot{x}(t)=-A x(t)+\sum_{i=1}^{n} B_{i} f_{k i}(x(t)),
$$

Based on Theorem 1, we can get the following corollary.

Corollary 2: Let $O=\left[0_{n}, 0_{n}, \ldots, 0_{n}\right]_{n, n n}$ and $B=\left[B_{1}, B_{2}, \ldots, B_{n}\right]_{n, n n}$. The system in (35) is asymptotically $N$-stable, if there exist matrices $P_{k}>0$, and diagonal matrices $S_{k i}^{+}$and $S_{k i}^{-}, k=1, \cdots, N, i=1, \cdots, n$, such that the following LMIs hold:

$$
\Theta_{k}=W_{P}^{T} \bar{P}_{k} W_{P}+\sum_{i=1}^{n}\left(W_{k S^{+}}^{T} \bar{S}_{k i}^{+} W_{k S^{+}}+W_{k S^{-}}^{T} \bar{S}_{k i}^{-} W_{k S^{-}}\right)<0,
$$


where

$$
\begin{aligned}
& \bar{P}_{k}=\left[\begin{array}{cc}
0_{n} & P_{k} \\
P_{k} & 0_{n}
\end{array}\right], \bar{S}_{k i}^{+}=\left[\begin{array}{cc}
0_{n} & S_{k i}^{+} \\
S_{k i}^{+} & -S_{k i}^{+}
\end{array}\right], \bar{S}_{k i}^{-}=\left[\begin{array}{cc}
0_{n} & S_{k i}^{-} \\
S_{k i}^{-} & -S_{k i}^{-}
\end{array}\right], W_{k P}=\left[\begin{array}{ccc}
-A & O & B \\
\hline I_{n} & O & O
\end{array}\right],
\end{aligned}
$$

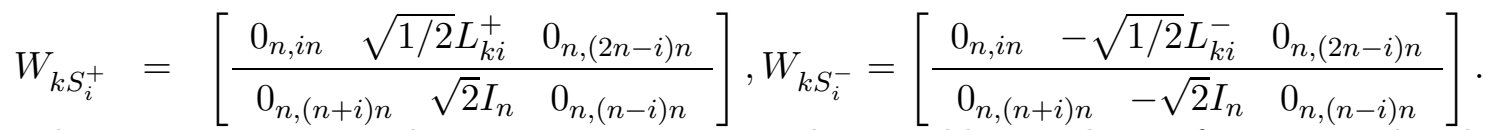

Remark 6: In our main results, we propose a general sector-like regulation function and utilize up-to-date techniques to derive stability conditions for GRNs for achieving delay dependence to ensure less conservatism. New conditions are derived for the general stability of a GRN in the form of linear matrix inequalities (LMIs) that are dependent on the upper and lower bounds of the delays. In the next section, the obtained general stability conditions are applied to several frequently used regulation functions. It is shown that the existing results for monostability of GRNs are special cases of our main results. Five examples are employed to illustrate the applicability and usefulness of the developed theoretical results.

\section{Illustrative Examples}

In this section, five examples are employed to show 1) the generality of out proposed regulation function; 2) the applicability of our main results for both the monostability and multistability; and 3) the more generality and less conservatism of our main results than existing ones.

Example 1 is concerned with a constructed GRN with five equilibrium points of which three are stable. Example 2 is concerned with a model of transcription factors (TFs) subjected to its own transcription regulation which is displaying bistability. Example 3 is concerned with a synthetic, bistable genetic toggle switch in Escherichia coli. Example 4 is concerned with a GRN with five nodes, and Example 5 is concerned with a synthetic oscillatory network of transcriptional regulators. It's shown that the existing results in Example 4 and Example 5 are special cases of our results.

Example 1: We consider a self regulatory network with one component:

$$
\dot{x}(t)=-a x(t)+f(x(t)),
$$

where $a$ denotes the decay rate of $x$ and $f(x)$ is the self regulation function. Let $f(x)=\tanh \left(x^{3}\right)$, which has a double sigmoid form, and $h(x)=\dot{f}(x)=3 x^{2}\left(1-\tanh ^{2}\left(x^{3}\right)\right) \leq 1.3432$. Both $f(x)$ and $h(x)$ are depicted in Fig. 3(b). Let $a=0.7$ and $g(x)=0.7 x$, so the cross points between $g(x)$ and $f(x)$ represent the equilibrium points of system (38). We can get five equilibrium points $x_{1}^{*}=-1.4, x_{2}^{*}=-0.9, x_{3}^{*}=0, x_{4}^{*}=0.9$ and $x_{5}^{*}=1.4$.

When $x_{1}^{*} \in(-\infty,-1], L_{1}^{-}=0, L_{1}^{+}=0.7384$, we can obtain a feasible solution by solving LMIs with the following obtained matrix variables:

$$
P_{1}=131.0213, S_{11}=137.1636, S_{12}=76.3891
$$

When $x_{2}^{*} \in[-1,-0.5], L_{2}^{-}=0.7384, L_{2}^{+}=1.518$, the solution is infeasible.

When $x_{3}^{*} \in[-0.5,0.5], L_{3}^{-}=0, L_{3}^{+}=0.7384$, we can obtain a feasible solution by solving LMIs with the following obtained matrix variables:

$$
P_{2}=131.0213, S_{21}=137.1636, S_{22}=76.3891
$$

When $x_{4}^{*} \in[0.5,1], L_{4}^{-}=0.7384, L_{4}^{+}=1.518$, the solution is infeasible.

When $x_{5}^{*} \in[1,+\infty), L_{5}^{-}=0, L_{5}^{+}=0.7384$, we can obtain a feasible solution by solving LMIs with the following obtained matrix variables:

$$
P_{3}=131.0213, S_{31}=137.1636, S_{32}=76.3891 .
$$


(a)

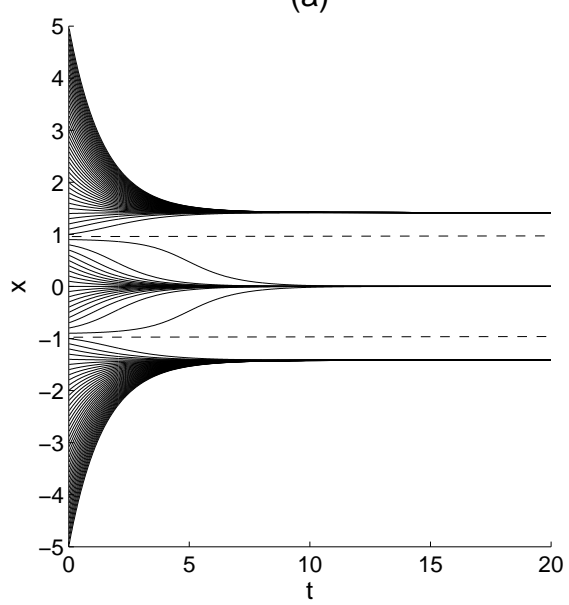

(b)

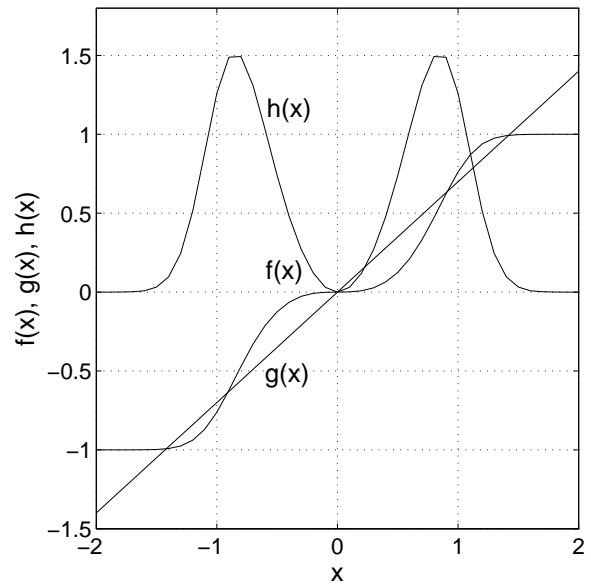

Fig. 3. (a) Transient behavior of system (38). (b) $f(x)=\tanh \left(x^{3}\right), h(x)=3 x^{2}\left(1-\tanh ^{2}\left(x^{3}\right)\right)$ and $g(x)=0.7 x$. Five equilibrium points can be obtained.

Simulation results are depicted in Fig. 3(a). The solid lines represent the stable states and the dashed lines represent the unstable states. It is concluded that the system (38) is 3 -stable.

Example 2: In [36], a relatively simple model of transcription factors (TFs) subject to positive and negative autoregulation of their own transcription is considered. By simplifications, a model with a single ordinary differential equation for the concentration of TF-A is given by

$$
\frac{\mathrm{d}[T F-A]}{\mathrm{d} t}=\frac{k_{1, f}[T F-A]^{2}}{[T F-A]^{2}+K_{1, d}}-k_{1, d}[T F-A]+r_{1, b a s}
$$

Parameter $k_{1, f}$ is the maximal rate of TF-A synthesis, $K_{1, d}$ is the dissociation constant of TF-A dimer from TF-REs, $k_{1, d}$ is the degradation rate constant of TF-A and $r_{1, b a s}$ is the basal rate of synthesis of activator. The regulation function is given by $f(x)=x^{2} /\left(K_{1, d}+x^{2}\right)$. The parameters values are given as $K_{1, d}=10$, $k_{1, d}=1 \mathrm{~min}^{-1}, r_{1, \text { bas }}=0.1 \mathrm{~min}^{-1}$. As shown in Fig. 2 in [36], the value of $k_{1, f}$ determines the type of general stability. For $6.1 \mathrm{~min}^{-1}<k_{1, f}<25.1 \mathrm{~min}^{-1}$, two stable state solutions for $[T F-A]$ exist. For $k_{1, f}<6.1$ $\min ^{-1}$ or $k_{1, f}>25.1 \mathrm{~min}^{-1}$, a unique stable state solution for $[T F-A]$ exists. Then system (39) can be rewritten into the following compact matrix form

$$
\dot{x}=-A x+B f_{k}(x)
$$

where

$$
\begin{aligned}
A & =k_{1, d}, B_{1}=k_{1, f}, \\
x(t) & =[T F-A]-[T F-A]_{k}^{*}, \\
f_{k}(x) & =f\left(x+[T F-A]_{k}^{*}\right)-f\left([T F-A]_{k}^{*}\right),
\end{aligned}
$$

and $f(x)$ and $\dot{f}(x)$ are shown in Fig. 4 .

Case 1: By taking $k_{1, f}=10 \mathrm{~min}^{-1}$, we can get three equilibrium points $x_{1}^{*}=0.138, x_{2}^{*}=0.99$ and $x_{3}^{*}=9.1$.

When $x_{1}^{*} \in[0,0.5], L_{1}^{-}=0, L_{1}^{+}=0.0952$, we can obtain a feasible solution by solving LMIs with the following obtained matrix variables:

$$
P_{1}=0.0278, S_{11}=0.7387, S_{12}=0.4189 .
$$

When $x_{2}^{*} \in[0.5,4], L_{2}^{-}=0.1183, L_{2}^{+}=0.2054$, the solution is infeasible. 


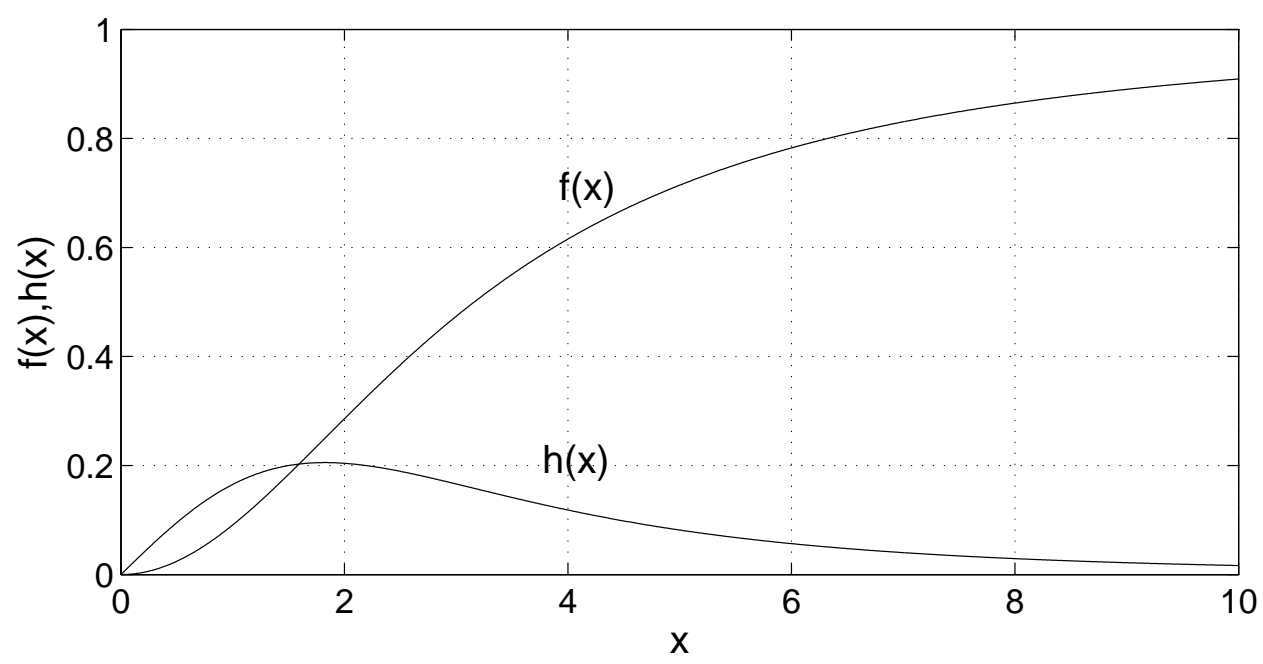

Fig. 4. $f(x)=x^{2} /\left(10+x^{2}\right)$, and $h(x)=\dot{f}(x)=20 x /\left(10+x^{2}\right)^{2}$.

When $x_{3}^{*} \in[4,+\infty), L_{3}^{-}=0, L_{3}^{+}=0.1183$, we can obtain a feasible solution by solving LMIs with the following obtained matrix variables:

$$
P_{2}=0.0264, S_{21}=0.7527, S_{22}=0.3685 \text {. }
$$

Case 2: By taking $k_{1, f}=1 \mathrm{~min}^{-1}$, we can get a unique equilibrium point $x_{1}^{*}=0.1055$.

When $x_{1}^{*} \in[0,+\infty), L_{1}^{-}=0, L_{1}^{+}=0.2054$, we can obtain a feasible solution by solving LMIs with the following obtained matrix variables:

$$
P_{1}=0.0230, S_{11}=0.7828, S_{12}=0.2266 \text {. }
$$

Case 3: By taking $k_{1, f}=30 \mathrm{~min}^{-1}$, we can get a unique equilibrium point $x_{1}^{*}=29.78$.

When $x_{1}^{*} \in[0,+\infty), L_{1}^{-}=0, L_{1}^{+}=0.2054$, we can obtain a feasible solution by solving LMIs with the following obtained matrix variables:

$$
P_{1}=0.3910, S_{11}=146.0866, S_{12}=10.2986
$$

Example 3: We consider a genetic toggle switch with bistability [4]:

$$
\begin{aligned}
& \dot{u}(t)=\frac{\alpha_{1}}{1+v^{\theta}\left(t-\tau_{v}\right)}-\beta_{1} u(t), \\
& \dot{v}(t)=\frac{\alpha_{2}}{1+u^{\gamma}\left(t-\tau_{u}\right)}-\beta_{2} v(t),
\end{aligned}
$$

where $u$ is the concentration of repressor 1, usually lacI, $v$ is the concentration of repressor 2 , usually cI, $\alpha_{1}$ is the effective rate of synthesis of repressor $1, \alpha_{2}$ is the effective rate of synthesis of repressor $2, \theta$ is the cooperativity of repression of promoter $2, \gamma$ is the cooperativity of repression of promoter $1, \beta_{1}$ is the ratio of the decay rate of repressor 1 and $\beta_{2}$ is the ratio of the decay rate of repressor 2 . We take the regulation function as $f(x)=x^{2} /\left(1+x^{2}\right)$, then we get the standard form:

$$
\begin{aligned}
& \dot{u}(t)=-\beta_{1} u(t)-\frac{\alpha_{1} v^{\theta}\left(t-\tau_{v}\right)}{1+v^{\theta}\left(t-\tau_{v}\right)}+r_{1, \text { bas }}, \\
& \dot{v}(t)=-\beta_{2} v(t)-\frac{\alpha_{2} u^{\gamma}\left(t-\tau_{u}\right)}{1+u^{\gamma}\left(t-\tau_{u}\right)}+r_{2, b a s},
\end{aligned}
$$


Then, system (41) can be rewritten into the following compact matrix form

$$
\dot{x}(t)=-A x(t)+\sum_{i=1}^{2} B_{i} f_{k}\left(x\left(t-\tau_{i}\right)\right)
$$

where

$$
\begin{aligned}
\tau_{1} & =\tau_{v}, \tau_{2}=\tau_{u}, \\
x(t) & =\left[\begin{array}{l}
x_{1}(t) \\
x_{2}(t)
\end{array}\right]=\left[\begin{array}{l}
u(t)-u_{k}^{*} \\
v(t)-v_{k}^{*}
\end{array}\right], A=\left[\begin{array}{cc}
\beta_{1} & 0 \\
0 & \beta_{2}
\end{array}\right], \\
B_{1} & =\left[\begin{array}{cc}
0 & -\alpha_{1} \\
0 & 0
\end{array}\right], B_{2}=\left[\begin{array}{cc}
0 & 0 \\
-\alpha_{2} & 0
\end{array}\right] \\
f_{k}\left(x\left(t-\tau_{i}\right)\right) & =\left[\begin{array}{c}
f_{k}\left(x_{1}\left(t-\tau_{i}\right)\right) \\
f_{k}\left(x_{2}\left(t-\tau_{i}\right)\right)
\end{array}\right]=\left[\begin{array}{c}
f\left(x_{1}\left(t-\tau_{i}\right)+u_{k}^{*}\right)-f\left(u_{k}^{*}\right) \\
f\left(x_{2}\left(t-\tau_{i}\right)+v_{k}^{*}\right)-f\left(v_{k}^{*}\right)
\end{array}\right],
\end{aligned}
$$

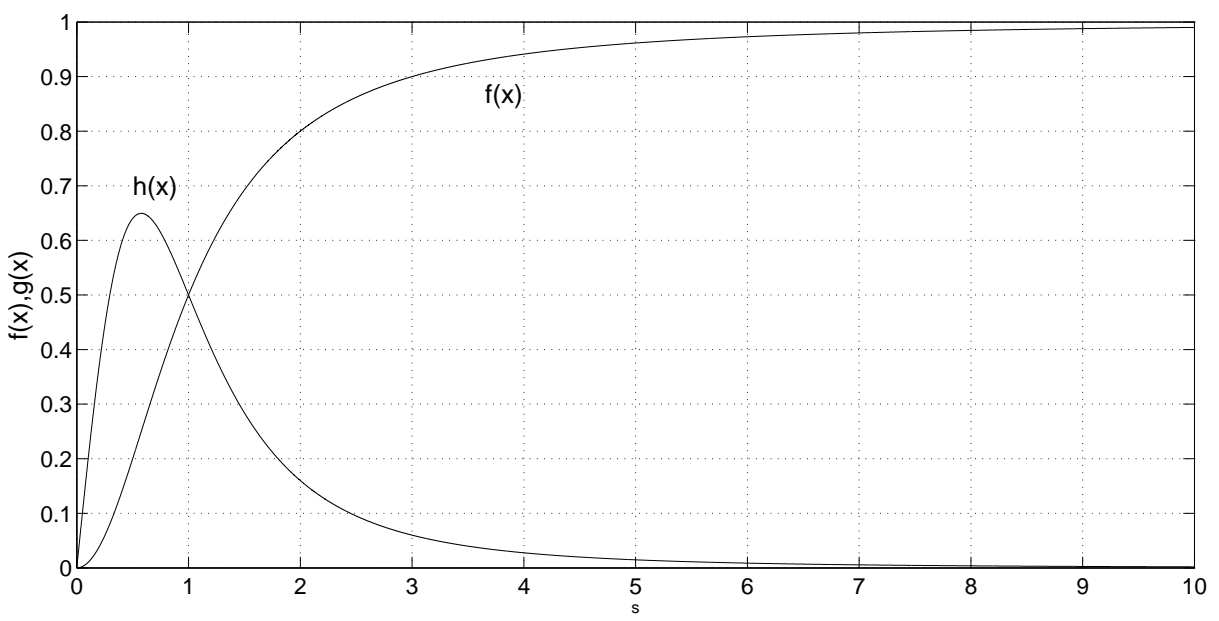

Fig. 5. $f(x)=x^{2} /\left(1+x^{2}\right)$, and $h(x)=\dot{f}(x)=2 x /\left(1+x^{2}\right)^{2}$.

We select a set of biologically plausible parameters as $\alpha_{1}=20, \alpha_{2}=20, \beta_{1}=\beta_{1}=1, \theta=\gamma=2$, $\tau_{1}=0.1$ and $\tau_{2}=0.2$. We can get three equilibrium points $x_{1}^{*}=(0.061,19.95)^{T}, x_{2}^{*}=(19.91,0.075)^{T}$ and $x_{3}^{*}=(2.59,2.43)^{T}$. The regulation function $f(x)$ and $\dot{f}(x)$ are shown in Fig. 5.

When $x_{1}^{*} \in[0,1.5] \times[1.5,+\infty), L_{1}^{-}=\operatorname{diag}\{0,0\}, L_{1}^{+}=\operatorname{diag}\{0.6495,0.06\}$. We can obtain a feasible solution by solving LMIs with the following obtained matrix variables (for space consideration, we only list the matrix variables $P_{1}, Z_{11}$ and $\left.Q_{11}\right)$ :

$$
P_{1}=\left[\begin{array}{ll}
0.0599 & 0.0170 \\
0.0170 & 0.0512
\end{array}\right], Z_{11}=\left[\begin{array}{cc}
0.2594 & -0.0006 \\
-0.0006 & 0.0568
\end{array}\right], Q_{11}=\left[\begin{array}{cc}
0.1897 & 0.0482 \\
0.0482 & 0.1738
\end{array}\right]
$$

When $x_{2}^{*} \in[1.5,+\infty) \times[0,1.5], L_{2}^{-}=\operatorname{diag}\{0,0\}, L_{2}^{+}=\operatorname{diag}\{0.06,0.6495\}$. We can obtain a feasible solution by solving LMIs with the following obtained matrix variables (for space consideration, we only list the matrix variables $P_{2}, Z_{21}$ and $Q_{21}$ ):

$$
P_{2}=\left[\begin{array}{ll}
0.0512 & 0.0170 \\
0.0170 & 0.0599
\end{array}\right], Z_{21}=\left[\begin{array}{ll}
0.2306 & 0.0001 \\
0.0001 & 0.2639
\end{array}\right], Q_{21}=\left[\begin{array}{ll}
0.1738 & 0.0508 \\
0.0508 & 0.1895
\end{array}\right] .
$$


When $x_{3}^{*} \in[1.5,3] \times[1.5,3], L_{3}^{-}=\operatorname{diag}\{0.06,0.06\}, L_{3}^{+}=\operatorname{diag}\{0.284,0.284\}$. The solution is infeasible.

Simulation results are depicted in Fig. 6. The solid lines represent the stable states and the dashed line represents the unstable states. So the genetic toggle switch (40) is bistable.

(a)

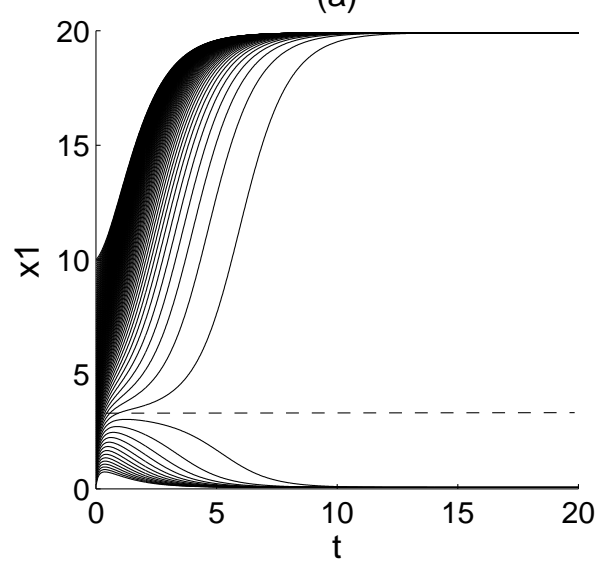

(b)

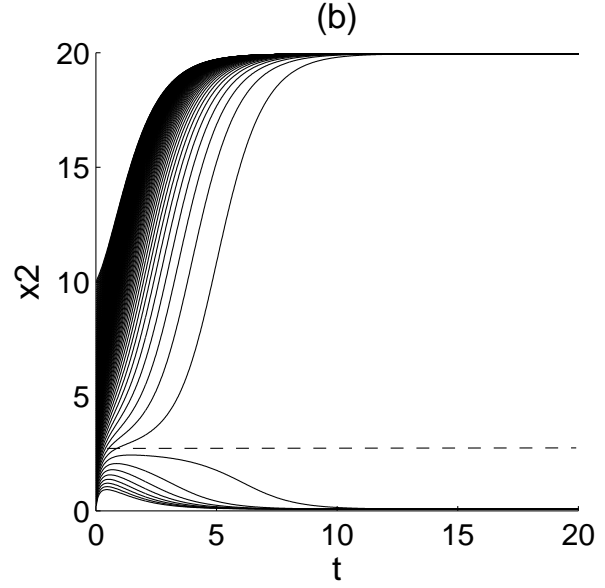

Fig. 6. Transient behavior of system (40), where $\alpha_{1}=20, \alpha_{2}=20$.

Example 4: Consider a small size genetic network with five nodes. In [9], the GRNs with time-varying delays are modeled as

$$
\begin{aligned}
\dot{m}_{i}(t) & =-a_{i} m_{i}(t)+\sum_{j} G_{i j} f\left(p_{j}\left(t-h_{1}(t)\right)\right), \\
\dot{p}_{i}(t) & =-c_{i} p_{i}(t)+d_{i} m_{i}\left(t-h_{2}(t)\right)
\end{aligned}
$$

where $a_{i}>0, c_{i}>0, i=1,2, \cdots, n, f(\cdot)$ is the regulation function with the same form described in Remark 1 , and $G$ is the coupling matrix of this GRN, which is defined as follows: if there is no link from node $i$ to node $j, G_{i j}=0$; if transcription factor $i$ is an activator of gene $j, G_{i j}=\alpha_{i j}>0$; if transcription factor $i$ is a repressor of gene $j, G_{i j}=-\alpha_{i j}<0$. In other words, the matrix $G=\left[G_{i j}\right]_{n, n}$ defines the coupling topology, direction, and the transcriptional rate of the genetic network. In compact matrix form, (42) and (43) can be rewritten as

$$
\begin{aligned}
\dot{m}(t) & =E m(t)+G f\left(p\left(t-h_{1}(t)\right)\right), \\
\dot{p}(t) & =C p(t)+D m\left(t-h_{2}(t)\right),
\end{aligned}
$$

Consider Remark $3, m(\cdot)$ in $(45)$ can be treated as a regulation function. This GRN is rewritten as

$$
\dot{x}(t)=A x(t)+\sum_{i=1}^{2} B_{i} g\left(x\left(t-h_{i}(t)\right)\right),
$$

where

$$
\begin{gathered}
x(t)=\left[\begin{array}{c}
m(t) \\
p(t)
\end{array}\right], g\left(x\left(t-h_{i}(t)\right)\right)=\left[\begin{array}{c}
m\left(t-h_{i}(t)\right) \\
f\left(p\left(t-h_{i}(t)\right)\right)
\end{array}\right], \\
A=\left[\begin{array}{ll}
E & 0 \\
0 & C
\end{array}\right], B_{1}=\left[\begin{array}{cc}
0 & G \\
0 & 0
\end{array}\right], B_{2}=\left[\begin{array}{cc}
0 & 0 \\
D & 0
\end{array}\right] .
\end{gathered}
$$

In order to test the effectiveness our theorem, all the parameters considered here are the same with [9]. Let 
the coupling matrix $G$ of this GRN as

$$
G=0.5 \times\left[\begin{array}{ccccc}
0 & -1 & 1 & 0 & 0 \\
-1 & 0 & 0 & 1 & 1 \\
0 & 1 & 0 & 0 & 0 \\
1 & -1 & 0 & 0 & 0 \\
0 & 0 & 0 & 1 & 0
\end{array}\right]
$$

and the system matrices be given as follows:

$$
E=C=\operatorname{diag}\{-1,-1,-1,-1,-1\}, \mathrm{D}=\operatorname{diag}\{0.8,0.8,0.8,0.8,0.8\} .
$$

In addition, the regulation function is given by

$$
g(p)=\frac{p^{2}}{1+p^{2}}
$$

and

$$
g(m)=m \text {. }
$$

It is easy to know $\dot{g}(p) \in[0,0.65]$ and $\dot{g}(m) \equiv 1 \in[1,1]$. Then we take $L^{-}=\operatorname{diag}\{1,1,1,1,1,0,0,0,0,0\}$ and $L^{+}=\operatorname{diag}\{1,1,1,1,1,0.65,0.65,0.65,0.65,0.65\}$. The time delays are assumed to be

$$
h_{1}(t)=1+0.1 \sin t, h_{2}(t)=0.5+0.1 \sin t
$$

and therefore we can get the parameters as follows :

$$
\begin{aligned}
& h_{11}=0.9, h_{21}=1.1, \mu_{1}=0.1 \\
& h_{12}=0.4, h_{22}=0.6, \mu_{2}=0.1
\end{aligned}
$$

We can obtain a feasible solution by solving LMIs with the following obtained matrix variables (for space consideration, we only list the matrix variables $\left.P_{1}\right)$ :

$$
P_{1}=\left[\begin{array}{rrrrrrrrrr}
4.8444 & 0.3277 & 0.4077 & -0.2195 & -0.4540 & -0.0731 & 0.0206 & 0.1570 & -0.0392 & -0.0370 \\
0.3277 & 4.6659 & 0.0798 & -0.0078 & -0.0564 & -0.1016 & -0.0219 & 0.0358 & 0.3109 & 0.0822 \\
0.4077 & 0.0798 & 5.7218 & 0.3525 & 0.3756 & -0.2863 & -0.1511 & 0.3815 & 0.2058 & -0.1058 \\
-0.2195 & -0.0078 & 0.3525 & 5.9240 & 0.4083 & 0.1789 & 0.2098 & 0.1802 & 0.2139 & 0.3269 \\
-0.4540 & -0.0564 & 0.3756 & 0.4083 & 5.1564 & -0.4010 & -0.0846 & 0.0584 & -0.3340 & 0.0039 \\
-0.0731 & -0.1016 & -0.2863 & 0.1789 & -0.4010 & 3.7020 & 0.0924 & 0.1319 & 0.4522 & 0.2133 \\
0.0206 & -0.0219 & -0.1511 & 0.2098 & -0.0846 & 0.0924 & 3.2710 & 0.0317 & 0.0415 & -0.0081 \\
0.1570 & 0.0358 & 0.3815 & 0.1802 & 0.0584 & 0.1319 & 0.0317 & 3.8921 & 0.1010 & 0.2110 \\
-0.0392 & 0.3109 & 0.2058 & 0.2139 & -0.3340 & 0.4522 & 0.0415 & 0.1010 & 3.8081 & 0.2326 \\
-0.0370 & 0.0822 & -0.1058 & 0.3269 & 0.0039 & 0.2133 & -0.0081 & 0.2110 & 0.2326 & 3.1573
\end{array}\right]
$$

To test less conservatism, the time delays are assumed to be

$$
h_{1}(t)=0.3+0.2 \sin 6 t, h_{2}(t)=1+0.5 \sin 3 t,
$$

and therefore we can get the parameters as follows:

$$
\begin{aligned}
& h_{11}=0.1, h_{21}=0.5, \mu_{1}=1.2, \\
& h_{12}=0.5, h_{22}=1.5, \mu_{2}=1.5 .
\end{aligned}
$$


We can obtain a feasible solution by solving LMIs with the following obtained matrix variables (for space consideration, we only list the matrix variables $\left.P_{1}\right)$ :

$$
P_{1}=\left[\begin{array}{rrrrrrrrrr}
4.7239 & 0.0351 & 4.5516 & -0.1302 & -0.1065 & -0.1805 & 0.0444 & -0.6841 & 0.2573 & 0.0415 \\
0.0351 & 1.8277 & 1.9968 & 1.7884 & -1.2289 & -0.0021 & -0.0818 & -0.2026 & -0.1021 & 0.0728 \\
4.5516 & 1.9968 & 16.0953 & 7.7194 & -1.7589 & 0.0082 & -0.1601 & -1.1333 & -0.3983 & 0.1970 \\
-0.1302 & 1.7884 & 7.7194 & 7.7071 & -1.0349 & 0.0956 & -0.1035 & -0.3483 & -0.7574 & 0.1604 \\
-0.1065 & -1.2289 & -1.7589 & -1.0349 & 6.2959 & 0.0262 & 0.0669 & 0.0282 & -0.1861 & -0.0163 \\
-0.1805 & -0.0021 & 0.0082 & 0.0956 & 0.0262 & 1.6371 & -0.0301 & 0.5879 & -0.1865 & 0.0985 \\
0.0444 & -0.0818 & -0.1601 & -0.1035 & 0.0669 & -0.0301 & 0.2440 & -0.0678 & 0.0246 & -0.0343 \\
-0.6841 & -0.2026 & -1.1333 & -0.3483 & 0.0282 & 0.5879 & -0.0678 & 4.9678 & 1.5075 & -0.2917 \\
0.2573 & -0.1021 & -0.3983 & -0.7574 & -0.1861 & -0.1865 & 0.0246 & 1.5075 & 2.4726 & -0.3472 \\
0.0415 & 0.0728 & 0.1970 & 0.1604 & -0.0163 & 0.0985 & -0.0343 & -0.2917 & -0.3472 & 0.0994
\end{array}\right],
$$

so this GRN is monostable.

Example 5: Consider a synthetic oscillatory network of transcriptional regulators. This network has been adopted as the mathematical model of the repressilator, which has been experimentally investigated in [28]. In this model, three repressor-protein concentrations, $p_{i}$, and their corresponding mRNA concentrations, $m_{i}$ (where $i$ is $l a c l$, tet $R$ or $c l$ ) are treated as continuous dynamical variables. Each of these six molecular species participates in transcription, translation, and degradation reactions. Here we consider only the symmetrical case in which all three repressors are identical except for their DNA-binding specificities. This GRN is also modeled by (42) and (43), we transform it into (46).

In order to test the effectiveness our theorem, all the parameters considered here are the same with [10]. Let the coupling matrix $G$ of this GRN as

$$
G=0.5 \times\left[\begin{array}{ccc}
0 & -1 & 1 \\
1 & 0 & -1 \\
-1 & 1 & 0
\end{array}\right]
$$

and the system matrices be given as follows:

$$
E=C=\operatorname{diag}\{-1,-1,-1\}, \mathrm{D}=\operatorname{diag}\{1,1,1\} .
$$

In addition, the regulation function is given by

$$
f(x)=\frac{x^{2}}{1+x^{2}} .
$$

It is easy to know $\dot{f}(x) \in[0,0.65]$ and $\dot{m} \in[1,1]$. Then we take $L^{-}=\operatorname{diag}\{1,1,1,0,0,0\}$ and $L^{+}=$ $\operatorname{diag}\{1,1,1,0.65,0.65,0.65\}$. The time delays are assumed to be

$$
\begin{aligned}
& h_{11}=0.9999, h_{21}=1, \mu_{1}=0, \\
& h_{12}=0.29999, h_{22}=0.3, \mu_{2}=0 .
\end{aligned}
$$

We can obtain a feasible solution by solving LMIs with the following obtained matrix variables (for space consideration, we only list the matrix variables $P_{1}$ ):

$$
P_{1}=\left[\begin{array}{rrrrrr}
1.6345 & 0.0362 & 0.0362 & -0.0061 & 0.0151 & 0.0234 \\
0.0362 & 1.6345 & 0.0362 & 0.0234 & -0.0061 & 0.0151 \\
0.0362 & 0.0362 & 1.6345 & 0.0151 & 0.0234 & -0.0061 \\
-0.0061 & 0.0234 & 0.0151 & 1.0620 & 0.0086 & 0.0086 \\
0.0151 & -0.0061 & 0.0234 & 0.0086 & 1.0620 & 0.0086 \\
0.0234 & 0.0151 & -0.0061 & 0.0086 & 0.0086 & 1.0620
\end{array}\right],
$$


To test less conservatism, the time delays are assumed to be

$$
h_{1}(t)=0.7+0.4 \sin 5 t, h_{2}(t)=1.5+0.5 \sin 4 t
$$

and therefore we can get the parameters as follows:

$$
\begin{aligned}
& h_{11}=0.3, h_{21}=1.1, \mu_{1}=2, \\
& h_{12}=1, h_{22}=2, \mu_{2}=2 .
\end{aligned}
$$

We can obtain a feasible solution by solving LMIs with the following obtained matrix variables (for space consideration, we only list the matrix variables $\left.P_{1}\right)$ :

$$
P_{1}=\left[\begin{array}{rrrrrr}
14.2180 & 0.1725 & 0.1725 & -0.3535 & 0.1508 & -0.1460 \\
0.1725 & 14.2180 & 0.1725 & -0.1460 & -0.3535 & 0.1508 \\
0.1725 & 0.1725 & 14.2180 & 0.1508 & -0.1460 & -0.3535 \\
-0.3535 & -0.1460 & 0.1508 & 4.0239 & 0.0061 & 0.0061 \\
0.1508 & -0.3535 & -0.1460 & 0.0061 & 4.0239 & 0.0061 \\
-0.1460 & 0.1508 & -0.3535 & 0.0061 & 0.0061 & 4.0239
\end{array}\right]
$$

and it follows that this GRN is monostable. We like to mention that, comparing to the results of [10], we no longer need the derivartives of the delays are less 1 , and therefore our results are less conservative.

\section{Conclusion}

In this paper, we have made an effort to show the possibility of applying control theory to investigate the general stability (monostability and multistability) of a GRN, therefore having potential applications in synthesis biology and system biology. A method has been presented for the analysis of general stability of a GRN with multiple time-varying delays and different types of regulation functions. By using a Lyapunov functional approach and linear matrix inequalities (LMI) techniques, the general stability criteria for a GRN with time-varying delays have been established in the form of LMIs, which can be readily verified by using standard numerical software. An important feature of the results reported here is that all the general stability conditions are dependent on the upper and lower bounds of the delays, which is made possible by utilizing the most updated techniques for achieving delay dependence. To the best of our knowledge, the approach presented here is the first computational approach developed specifically for general stability of a GRN. Five examples have been employed to illustrate the applicability and usefulness of the developed theoretical results, which are concerned with, respectively, a constructed GRN with five equilibrium points of which three are stable, a model of transcription factors (TFs) subjected to its own transcription regulation which is displaying bistability, a synthetic bistable genetic toggle switch in Escherichia coli, a GRN with five nodes and a synthetic oscillatory network of transcriptional regulators. It has been shown that our results are more general and less conservative than existing ones.

\section{REFERENCES}

[1] S. Benner and A. Sismour, Synthetic biology, Nat Rev Genet, vol. 6, no. 7, pp. 533.543, 2005.

[2] D. Endy, Foundations for engineering biology, Nature, vol. 438, no. 7067, pp. 449-453, 2005.

[3] E. Andrianantoandro, S. Basu, D. Karig, and R. Weiss, Synthetic biology: new engineering rules for an emerging discipline, Mol. Syst. Biol, vol. 2, no. 2006.0028, 2006.

[4] T. Gardner, C. Cantor, and J. Collins, Construction of a genetic toggle switch in Escherichia coli. Nature, vol. 403, no. 6767, pp. 339-42, 2000.

[5] C. Bagowski, J. Besser, C. Frey, and J. Ferrell Jr, The JNK Cascade as a Biochemical Switch in Mammalian Cells Ultrasensitive and All-or-None Responses, Current Biology, vol. 13, no. 4, pp. 315-320, 2003.

[6] N. Wiener, Cybernetics or Control and Communication in the Animal and the Machine. MIT Press, 1961. 
[7] C. Tomlin, Understanding biology by reverse engineering the control. Proceedings of the National Academy of Sciences, vol. 102, no. 12, pp. 4219-4220, 2005.

[8] A. Becskei and L. Serrano, Engineering stability in gene networks by autoregulation. Nature, vol. 405, no. 6786, pp. 590-3, 2000.

[9] C. Li, L. Chen, and K. Aihara, Stability of Genetic Networks With SUM Regulatory Logic: Lur'e System and LMI Approach, IEEE Transactions on Circuits and Systems I: Regular Papers, vol. 53, no. 11, pp. 2451-2458, 2006.

[10] Z. Wang, H. Gao, J. Cao, and X. Liu, On Delayed Genetic Regulatory Networks With Polytopic Uncertainties: Robust Stability Analysis, IEEE Transactions on NanoBioscience, vol. 7, no. 2, pp. 154-163, 2008.

[11] J. Cao and F. Ren, Exponential Stability of Discrete-Time Genetic Regulatory Networks With Delays, IEEE Transactions on Neural Networks, vol. 19, no. 3, pp. 520-523, 2008.

[12] J. Monod and F. Jacob, Teleonomic mechanisms in cellular metabolism, growth and differentiation, Cold Spring Harbor Symposia on Quantitative Biology, vol. 26, p. 389-401, 1961.

[13] E. Ozbudak, M. Thattai, H. Lim, B. Shraiman, and A. van Oudenaarden, Multistability in the lactose utilization network of Escherichia coli. Nature, vol. 427, no. 6976, pp. 737-740, 2004.

[14] J. Ferrell Jr and E. Machleder, The Biochemical Basis of an All-or-None Cell Fate Switch in Xenopus Oocytes. Science, vol. 280, no. 5365, p. 895, 1998.

[15] J. Pomerening, E. Sontag, and J. Ferrell Jr, .Building a cell cycle oscillator: hysteresis and bistability in the activation of Cdc 2, Nature Cell Biology, vol. 5, no. 4, pp. 346-351, 2003.

[16] M. Laurent and N. Kellershohn, Multistability: a major means of differentiation and evolution in biological systems, Trends in Biochemical Sciences, vol. 24, no. 11, pp. 418-422, 1999.

[17] D. Angeli and E. Sontag, Multi-stability in monotone input output systems, Systems \& Control Letters, vol. 51, no. 1-2, pp. 185-202, 2004.

[18] D. Angeli and E. Sontag, Monotone control systems, IEEE Transactions on Automatic Control, vol. 48, no. 10, pp. 1684-1698, 2003.

[19] M. Chaves, T. Eissing, and F. Allgower, Bistable Biological Systems: A Characterization Through Local Compact Input-toState Stability, IEEE Transactions on Automatic Control, vol. 53, no. 1, pp. 87-100, 2008.

[20] D. Angeli, J. Ferrell Jr, and E. Sontag, Detection of multistability, bifurcations, and hysteresis in a large class of biological positive-feedback systems. Proceedings of the National Academy of Sciences, vol. 101, no. 7, pp. 1822-1827, 2004.

[21] M. Savageau, Design principles for elementary gene circuits: Elements, methods, and examples. Chaos: An Interdisciplinary Journal of Nonlinear Science, vol. 11, p. 142, 2001.

[22] P. Smolen, D. Baxter, and J. Byrne, Mathematical Modeling of Gene Networks. Neuron, vol. 26, no. 3, pp. 567-580, 2000.

[23] H. de Jong, .Modeling and Simulation of Genetic Regulatory Systems: A Literature Review. Journal of Computational Biology, vol. 9, no. 1, pp. 67-103, 2002.

[24] G. Batt, B. Yordanov, R. Weiss, and C. Belta, Robustness analysis and tuning of synthetic gene networks. Bioinformatics, vol. 23, no. 18, p. 2415, 2007.

[25] S. Boyd, L. El Ghaoui, E. Feron, and V. Balakrishnan, Linear Matrix Inequalities in System and Control Theory. Society for Industrial Mathematics, 1994.

[26] C. Yuh, H. Bolouri, and E. Davidson, Genomic Cis-Regulatory Logic: Experimental and Computational Analysis of a Sea Urchin Gene. Science, vol. 279, no. 5358, p. 1896, 1998.

[27] J. Tyson and H. Othmer, The dynamics of feedback control circuits in biochemical pathways. Progress in theoretical biology, Vol. 5, 1-62, p. 5, 1978.

[28] M. Elowitz and S. Leibler, A synthetic oscillatory network of transcriptional regulators. Nature, vol. 403, no. 6767, pp. 335-8, 2000 .

[29] H. Gao and C. Wang, A delay-dependent approach to robust $H_{\infty}$ filtering for uncertain discrete-time state-delayed systems. IEEE Transactions on Signal Processing, vol. 52, no. 6, pp. 1631-1640, 2004.

[30] Z. Wang, D. W. C. Ho, Y. Liu and X. Liu, Robust $H_{\infty}$ control for a class of nonlinear discrete time-delay stochastic systems with missing measurements, Automatica, Vol. 45, No. 3, Mar. 2009, pp. 684-691.

[31] Z. Wang, Y. Liu and X. Liu, $H_{\infty}$ filtering for uncertain stochastic time-delay systems with sector-bounded nonlinearities, Automatica, Vol. 44, No. 5, May 2008, pp. 1268-1277.

[32] H. Gao and T. Chen, New Results on Stability of Discrete-Time Systems With Time-Varying State Delay, IEEE Transactions on Automatic Control, vol. 52, no. 2, pp. 328-334, 2007.

[33] Y. Liu, Z. Wang and X. Liu, Global exponential stability of generalized recurrent nueral networks with discrete and distributed delays. Neural Networks, vol. 19, no. 5, pp. 667-675, 2006.

[34] Z. Wang, H. Shu, Y. Liu, D.W.C. Ho and X. Liu, Robust stability analysis of generalized neural networks with discrete and distributed time delays. Chaos, Solitons and Fractals, vol. 30, no. 4, pp. 886-896, 2006.

[35] Z. Wang, Y. Liu and X. Liu, State estimation for jumping recurrent neural networks with discrete and distributed delays, Neural Networks, Vol. 22, No. 1, Jan. 2009, pp. 41-48. 
[36] P. Smolen, D. Baxter, and J. Byrne, Frequency selectivity, multistability, and oscillations emerge from models of genetic regulatory systems, American Journal of Physiology- Cell Physiology, vol. 274, no. 2, pp. 531-542, 1998.

[37] Z. Wang, X. Liu, Y. Liu, J. Liang and V. Vinciotti, An extended Kalman filtering approach to modelling nonlinear dynamic gene regulatory networks via short gene expression time series, IEEE/ACM Transactions on Computational Biology and Bioinformatics, Vol. 6, No. 3, Jul.-Sept. 2009, pp. 410-419.

[38] Z. Wang, J. Lam, G. Wei, K. Fraser and X. Liu, Filtering for nonlinear genetic regulatory networks with stochastic disturbances, IEEE Transactions on Automatic Control, Vol. 53, No. 10, Nov. 2008, pp. 2448-2457.

[39] Z. Wang, F. Yang, D. W. C. Ho, S. Swift, A. Tucker and X. Liu, Stochastic dynamic modeling of short gene expression time series data, IEEE Transactions on NanoBioscience, Vol. 7, No. 1, Mar. 2008, pp. 44-55. 